\title{
NIÑEZ E INTERPRETACIÓN AMBIENTAL EN EL PROYECTO JARDÍN BOTÁNICO DE LA UNIVERSIDAD DEL CAUCA ÁLVARO JOSÉ NEGRET (UCAJN)
}

\author{
MARITSA CRUZ MUÑOZ ${ }^{1}$, LADY D. CASTRO RAMÍREZ ${ }^{2}$ \\ $Y$ DIEGO JESÚS MACÍAS PINTO ${ }^{3}$ \\ macruz258@gmail.com
}

Manizales, 2010-10-15 (Rev. 2011-05-24)

\section{RESUMEN}

La evaluación cualitativa mediante la técnica de mapas conceptuales, realizada en el espacio Jardín Botánico-UCAJN con un grupo de niños de cuarto y quinto grado de las instituciones educativas: Comercial del Norte Francisco José Chaux Ferrer (CNFJCF) de la vereda La Rejoya y Rafael Pombo (RP) de la ciudad de Popayán a través de tres fases consecutivas (exploración, validación y aplicación), permitió evidenciar un aprendizaje significativo ascendente, reflejado en la asimilación progresiva de conceptos, tanto en la estructuración de los mapas conceptuales como en la adquisición de valores ético-ambientales (estético, ecológico, económico, humanístico, moral, recreativo y científico) hacia el entorno. A lo largo de la investigación, esta progresión condujo a estructurar una charla modelo de interpretación ambiental para niños en edad escolar y a soportar posteriormente el plan de interpretación ambiental para el proyecto de Jardín Botánico-UCAJN.

PALABRAS CLAVE: Interpretación ambiental, mapas conceptuales, aprendizaje significativo, jardín botánico, Cauca.

\section{CHILHOOD AND ENVIRONMENTAL INTERPRETATION IN THE UNIVERSIDAD DEL CAUCA ÁLVARO JOSÉ NEGRET BOTANICAL GARDEN PROJECT (UCAJN)}

\section{ABSTRACT}

The qualitative evaluation by means of the conceptual maps technique carried out in the UCAJN botanical garden space with a group of fourth and fifth grade children from the educational institutions North Commercial Chaux Francisco José Ferrer (CNFJCF) of La Rejoya and Rafael Pombo (RP) in the city of Popayán through three consecutive phases (exploration, validation and implementation), allowed to demonstrate an increasingly significant learning process reflected in the gradual assimilation of concepts, both in the structure of conceptual maps and the acquisition of ethical and environmental values (aesthetic, ecological, economic, humanistic, moral, recreational, and scientific) towards the environment. Throughout the investigation, this progression led to the structure of an environmental interpretation talk model for school children and then to support the environmental interpretation plan for the UCAJN Botanical Garden project.

KEY WORDS: environmental interpretation, conceptual maps, meaningful learning, botanical garden, Cauca.

\section{INTRODUCCIÓN}

Freeman Tilden, el padre de la interpretación ambiental (I.A.) la definió como el proceso de revelar las maravillas naturales culturales e históricas de un entorno dado y estableció seis principios para conducir una interpretación exitosa: la experiencia directa entre el visitante y el recurso interpretado, la interpretación como un arte, provocación y una forma de comunicación, la relación entre todos los elementos naturales del medio y el direccionamiento hacia audiencias específicas (Tilden, 1957). A lo largo del tiempo la I.A. se ha consolidado como una herramienta eficaz de educación ambiental informal para espacios y áreas protegidas (Morales, 1998), la difusión in situ y la puesta en valor de lugares de interés como parques naturales, yacimientos arqueológicos, sitios históricos, cascos urbanos y jardines botánicos (Morales,n.f.), siendo una disciplina que revela significados y relaciones mediante el uso de objetos originales (seres vivos e inertes), experiencias directas en diferentes áreas y medios ilustrativos, herramientas audiovisuales, maquetas, folletos y sonidos (Dawson, 1999).

En Colombia actualmente existen más de 25 jardines botánicos organizados en una Red Nacional, a través de la cual se han establecido los criterios de planificación y constitución para nuevos jardines en diversas áreas del país, con el fin de promover ampliamente la conservación, investigación, educación ambiental, comunicación y divulgación, así como los procesos administrativos que garanticen su funcionamiento (Samper y García, 2001a). Así, el Jardín Botánico-UCAJN es un 
proyecto naciente de ciencia y tecnología establecido mediante el convenio interinstitucional 073/1999, que busca generar identidad y valoración de la naturaleza en las comunidades, entidades públicas y privadas de la región caucana y del suroccidente colombiano, propiciando una zona de encuentro para el disfrute, la estética, la investigación, la educación, la conservación y el manejo de los recursos naturales existentes, contribuyendo al crecimiento social y cultural (Figueroa et al., 2002).

El Plan Nacional de Jardines Botánicos (2001) establece la importancia de la caracterización y el reconocimiento de audiencias potenciales (Samper y García, 2001b) y específicamente, la creación del programa nacional de educación ambiental para niños en jardines botánicos los cataloga como un grupo esencial (Sierra, 2004). Siguiendo estos preceptos y fundamentados además en el sexto principio de Tilden (1957): "la interpretación debe ir dirigida a una audiencia determinada, con un enfoque especial para niños", surge el interés por el análisis interpretativo con niños en el Jardín Botánico-UCAJN a través del establecimiento de interrelaciones entre mapas conceptuales y aprendizaje significativo, con el fin de evaluar las conexiones y percepciones de estos visitantes hacia el ambiente circundante (Macías et al., 1996).

Con la técnica de mapas conceptuales, los niños representaron estructuras conceptuales que permitieron guiar y evaluar el aprendizaje significativo, reflejado en la construcción de conceptos para entender las interrelaciones del medio (Talero y Umaña, 1993). Los mapas conceptuales fueron evaluados y valorados mediante la metodología de Novak y Gowin (1988), para determinar el nivel de asimilación de conceptos y valores ético-ambientales del estudiante hacia el Jardín Botánico-UCAJN; lo que contribuyó finalmente con la esencia misma de la interpretación ambiental: la conexión intelectual y emocional entre los intereses de la audiencia y el significado inherente del recurso (Knapp y Benton, 2004, p.10), siendo el resultado directo la charla interpretativa modelo para niños en edad escolar que visitaran este espacio.

La selección de las instituciones educativas, Rafael Pombo (RP) de la ciudad de Popayán (área urbana) y Comercial del Norte sede Francisco José Chaux Ferrer (CNFJCF) de la veredaa Rejoya (área rural), son el reflejo de los dos modelos de educación primaria en Colombia: la escuela urbana y la escuela nueva (rural).

La escuela nueva surge en la década de los setenta, como una estrategia para universalizar la educación primaria rural a través de un sistema integrado que combina cuatro aspectos: currículo, capacitación, administración y comunitario.

Básicamente la metodología se fundamenta en la realidad de la escuela multigrado, donde uno o más profesores están encargados de los cinco grados de enseñanza primaria, actuando como facilitadores (guiando, orientando y evaluando el aprendizaje), promotores y organizadores del trabajo directo con la comunidad (padres de familia), mientras que los niños estudian en pequeños grupos usando guías autodidácticas donadas por el Estado (Torres, 1992). La expansión de este programa en Colombia fue significativo, existiendo 17.948 escuelas con 800.000 estudiantes para el año 1989 esperando un cubrimiento total en los próximos años con 41.000 nuevas escuelas, catalogándose como una estrategia a seguir en otros países latinoamericanos (Psacharopoulos, Rojas \& Vélez, 1992).

Por su parte, la escuela urbana sigue los lineamientos de la escuela tradicional, reconocida según Gómez (2002) por los siguientes tres polos característicos: el conjunto en modos de actuar en pedagogía producto de una tradición, la referencia cultural y humanista al pasado, y el proceso formal de transmisión y de tradición.

\section{METODOLOGÍA}

El estudio se llevó a cabo en el Jardín Botánico-UCAJN (Figura 1), localizado en la vereda La Rejoya, ubicado a $18 \mathrm{~km}$ al occidente del casco urbano del municipio de Popayán, al nororiente del departamento del Cauca, entre los $2^{\circ} 31^{\prime} 13^{\prime \prime}$ de latitud norte y $76^{\circ} 35^{\prime} 46^{\prime \prime}$ de longitud oeste, con una extensión de 12,8 ha y con una altitud de 1600-1800 msnm.

Figura 1. Localización de la zona de estudio. 

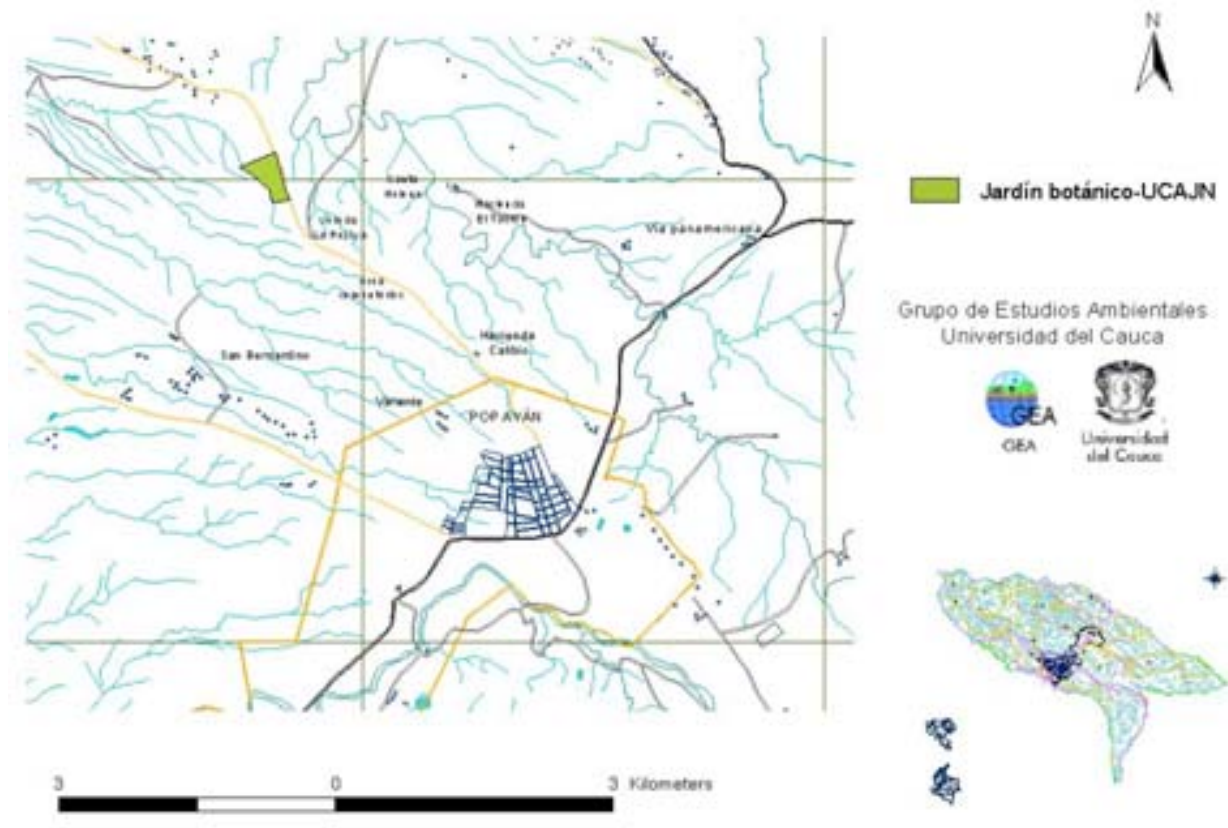

Cartogratia elaborada por Cruz, M. Castro, L. joaqui, S

Con el apoyo de maestros y directivas escolares, los grupos de cuarto y quinto entre 8-11 años (niñas y niños), de las instituciones educativas CNFJCF y RP, fueron seleccionados para llevar a cabo el análisis interpretativo, dada su capacidad lectora y escritura más desarrollada, al igual que su motricidad para desplazarse en el Jardín Botánico-UCAJN. Para ello se efectuaron los siguientes procedimientos:

\section{1) Capacitación para elaboración de mapas conceptuales}

Previo al desarrollo de las tres fases en el Jardín Botánico-UCAJN, los niños fueron capacitados en elaboración de mapas conceptuales siguiendo los planteamientos de Novak y Gowin (1988), utilizando ejemplos apropiados, muy relacionados con su entorno (familia, institución educativa, la naturaleza) tomando como base el texto escolar de básica primaria de Acevedo (2002).

\section{2) Preparación del recorrido}

Este proceso se estructuró siguiendo los planteamientos de Dawson (1999) y Macías, Sánchez y Ramírez (1999), en los que se establecen las medidas para la seguridad del recorrido, las instrucciones básicas que se darán durante el mismo, la presentación y las estrategias de observación.

\section{3) Recorridos en el jardín botánico}

En compañía de un grupo de estudiantes de la institución educativa RP y otro de de CNFJCF, se realizaron caminatas por las áreas del jardín botánico en tres fases: exploración, validación y aplicación.

\section{Fase de exploración}

Etapa donde los niños observaron, detallaron e investigaron el entorno. El papel de los guías estuvo limitado a la realización de una charla de orientación por las áreas previamente seleccionadas en el Jardín Botánico-UCAJN. En esta fase se observaron los intereses y se dio respuesta a las preguntas realizadas por los estudiantes durante el recorrido.

\section{Fase de validación}


Con base en las observaciones de la fase anterior, se diseñó una charla partiendo de las características, necesidades y debilidades de las nueve estaciones trabajadas con los niños en la primera fase. De esta manera, los temas propuestos estuvieron enfocados en: la importancia del Jardín Botánico-UCAJN para la comunidad local-regional, las plantas medicinales como recurso local, mantenimiento y valoración de las plantas, el jardín como ambiente de sano esparcimiento mediante recreación y entorno de expresión, relaciones ecológicas primordiales en selvas intervenidas, procesos orgánicos y principales características de ambientes áridos. En la realización de la charla se tuvo en cuenta la pertinencia del tiempo empleado (45 minutos) y la capacidad de mantener atentos a los escolares.

\section{Fase de aplicación}

Los temas de la fase de validación fueron soportados y cualificados según las características del jardín botánico y conociendo las necesidades y falencias (valor económico y ejemplos adecuados) reflejadas por los niños en los mapas conceptuales, en las fases anteriores, estableciendo un contraste directo con el recurso a interpretar. Esto permitió cualificar la charla buscando captar en gran medida la atención de los niños en el momento de evaluarla.

\section{4) Elaboración y evaluación de mapas conceptuales}

Una vez terminado el recorrido por el Jardín Botánico-UCAJN en cada fase, cada niño o niña elaboró un mapa conceptual del Jardín Botánico-UCAJN, tomando como base lo observado en el ambiente y la información suministrada por los orientadores en todas las estaciones. Posteriormente, los mapas fueron evaluados siguiendo la metodología de Talero y Umaña (1993), a través de los siguientes criterios:

- $\quad$ Nivel de jerarquía (NJ). Representa el orden de los conceptos en el mapa conceptual y su importancia como conceptos supraordenados o subordinados. El valor numérico asignado para cada NJ válido fue 3 .

- Relación válida (RV). Capacidad de entrelazar conceptos estrechamente relacionados, permitiendo clarificar el mapa conceptual. El valor numérico asignado para cada RV fue 1 , por ser un nivel de menor aprendizaje significativo.

- Relaciones cruzadas (RC). Proposiciones válidas entre dos conceptos distantes que aparentemente no tienen relación. El valor numérico asignado fue 6 , por ser reconciliaciones integradoras, que permiten constituir los conceptos existentes en un concepto integrador mayor.

- Ejemplos adecuados (EjA). Ejemplos usados por los niños al construir las relaciones y jerarquías. El valor asignado fue 0,5 por indicar la menor magnitud en el aprendizaje significativo.

La segunda parte de la evaluación fue el análisis valorativo de los mapas conceptuales, mediante la identificación de los valores ético-ambientales que cada niño debía reflejar para que la interacción con el medio ambiente circundante sea productiva y transformadora. Estos valores fueron: estético $(E)$, ecológico $(E C)$, económico $\left(E C^{\prime}\right)$, humanístico $(H)$, moral $(M)$, recreativo $(R)$ y científico $(C)$, según los planteamientos de Talero y Umaña (1993), quereconocen los valores de conservación (los valores económicos, humanistas, ecológicos y científicos) como fundamentales para el equilibrio dinámico del ambiente y sugieren que la acción educativa es una acción profundamente humanizadora, capaz de favorecer y de potenciar en los individuos el libre e interiorizado desarrollo de valores que les permitan conjugar, en armonía, el aprender a aprender y el aprender a vivir como dos realidades que se chocan y se funden en el proceso educativo.

Con los contenidos de todos los mapas conceptuales, se elaboró un mapa integrado del Jardín Botánico-UCAJN para cada fase, que sirvió para contrastar y evidenciar la evolución del conocimiento de los grupos escolares.

\section{5) Consolidación de la charla modelo}

Una vez aplicada la última fase, la evaluación y valoración de los mapas conceptuales permitió establecer la charla modelo de interpretación ambiental para niños en edad escolar, que sería la guía para futuras caminatas interpretativas dentro del Jardín Botánico-UCAJN, que entraría en transformación permanente debido a los cambios del ambiente.

\section{RESULTADOS}




\section{1) Evaluación y valoración progresiva de los mapas conceptuales a través de las tres fases}

La evaluación y valoración de los mapas conceptuales (Tablas 1 y 2), indica un aumento general progresivo en el aprendizaje significativo adquirido por los escolares (Figuras 2 y 3 , respectivamente), tanto en la destreza para elaboración como en los valores ético-ambientales desarrollados hacia el entorno. A medida que la charla interpretativa fue reestructurada a través de las diferentes fases (exploración, validación y aplicación), se incluyeron los conceptos y valores ausentes o poco evidenciados en la fase inmediatamente anterior.

\section{Evaluación de mapas conceptuales}

Se refleja la capacidad progresiva de los niños para establecer conceptos en orden jerárquico en las diferentes fases (Tablas 1 y 2); entre la primera y segunda fase se visualizó un incremento de 0,3 para la institución educativa CNFJCF y de 0,8 para RP; entre la segunda y la tercera fase el aumento fue de 4,6 y 3, respectivamente (Figura 2); lo que indica mayor asimilación de los conceptos (mayor número de niveles jerárquicos plasmados) a través de los recorridos.

Para las relaciones válidas, que indican la capacidad de los niños para relacionar y enlazar conceptos de forma apropiada aumentando el nivel de profundidad al captar el mensaje de la charla, el resultado fue aumentando en las diferentes fases; en la institución educativa CNFJCF el incremento fue de 1 entre la fases exploratoria-validación y de 4,6 entre las fases validaciónaplicación; los incrementos fueron de 6 y 2,4, respectivamente, para la institución RP (Figura 2).

Al visualizar las relaciones cruzadas, se puede evidenciar el progreso en la capacidad de los niños para establecer conexiones entre conceptos distantes que cumplen un tipo de relación ecológica intra e interespecífica en el medio. Estas relaciones son fundamentales para el aprendizaje significativo porque los niños adquieren la capacidad de interpretar, captar y profundizar conceptos sobre su entorno y relacionarlos con otros aspectos de su experiencia formando un conocimiento integral, que refleja el verdadero sentido de la educación ambiental. La destreza de los niños aumentó claramente al establecer relaciones cruzadas en los mapas conceptuales, observándose un incremento de 1,2 para la institución CNFJCF y 1,9 para RP entre las fases exploratoriavalidación y 4,2 y 7,4 entre las fases de validación-aplicación, respectivamente (Figura 2).

Aunque la capacidad de los niños, para realizar ejemplos acordes a cada concepto dado en cada una de las charlas, se tornó oscilatoria a través de las diferentes fases, existió un progreso de 0,3 en la institución educativa CNFJCF y 1,6 en RP entre las fases exploratoria-validación. Entre las fases de validación-aplicación hubo una disminución de 0,2 y 1,7 para cada una de las instituciones respectivamente (Figura 2); incluyendo nuevos ejemplos en las zonas del Jardín Botánico-UCAJN en la charla modelo estructurada, aunque representan el mínimo valor en el aprendizaje significativo. 
Figura 2. Evaluación de mapas conceptuales.

Institución educativa CNFJCF

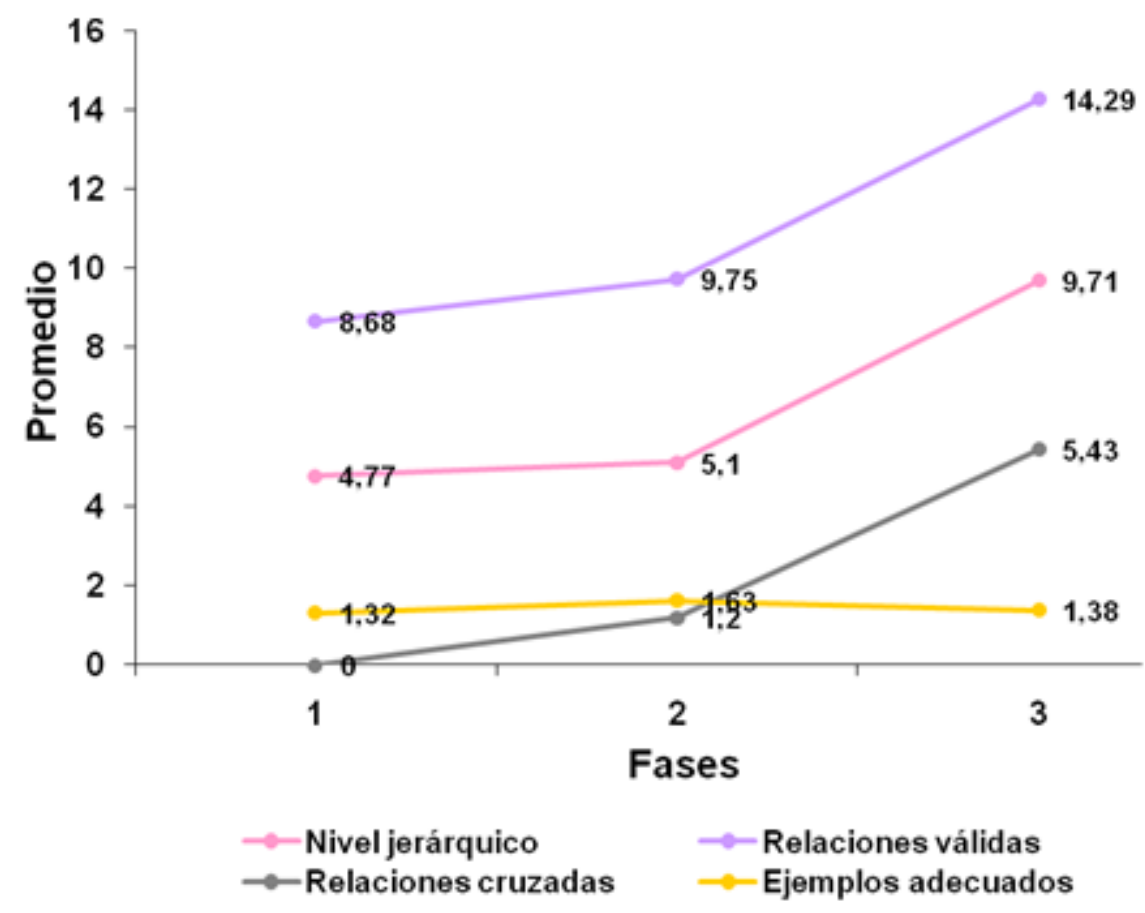

Institución educativa RP

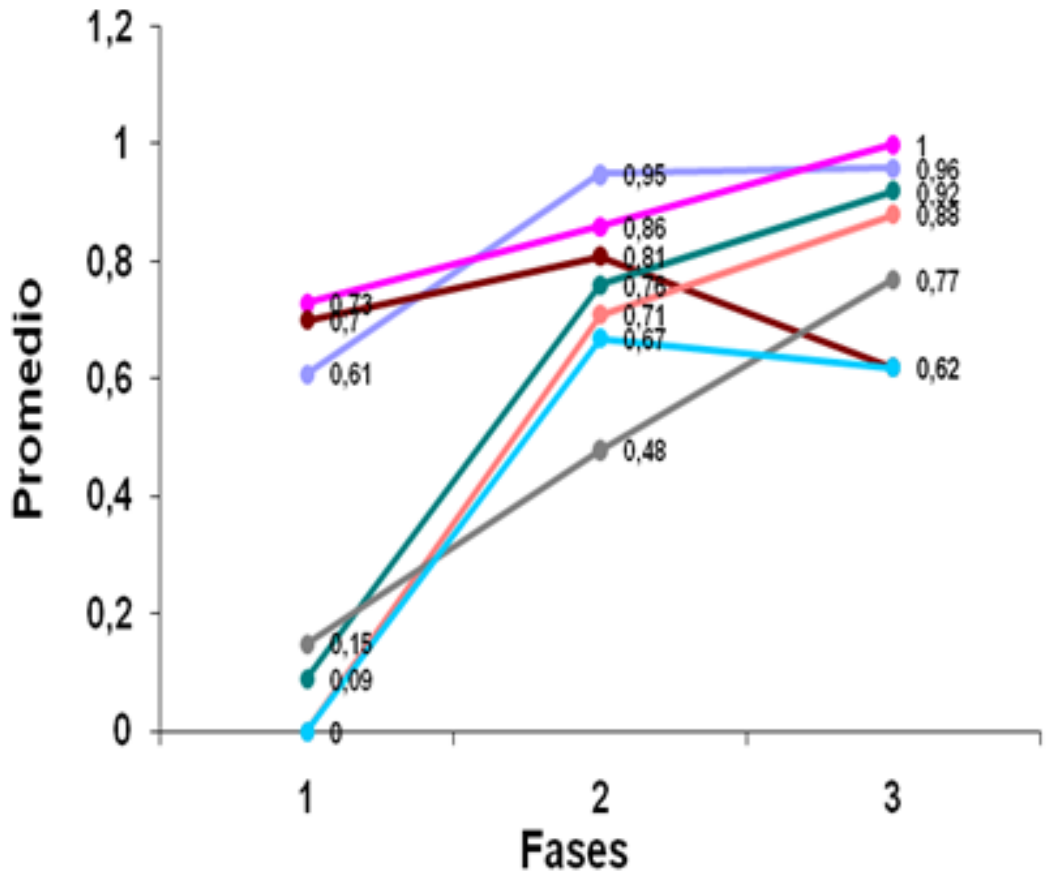

$\rightarrow \rightarrow$ Estético $\rightarrow$-Ecológico $\rightarrow$-Económico $\rightarrow$-Humanistico




\section{Valoración de mapas conceptuales}

Cuando un estudiante plasma entre otros aspectos, los diferentes valores éticos (de tipo estético, ecológico, económico, humanístico, moral, recreativo y científico) en el mapa conceptual, refleja el nivel de asimilación de los contenidos y su capacidad de percepción y entendimiento; aspectos relevantes que, se pretende, perduren en la mente de los niños y sean reflejados en su vida cotidiana. Al observar los valores éticos reflejados en los mapas conceptuales de los niños de las dos instituciones educativas (Tablas 1 y 2), se puede notar una progresión constante de la mayoría de ellos, aunque se presentó una declinación marcada del valor económico y otras reducciones leves en los valores moral y recreativo para la institución educativa CNFJCF, y un descenso elevado en el valor económico y una reducción muy baja para el valor científico para la institución educativa RP (Figura 3). Esto evidencia la necesidad de enfatizar sobre conceptos y/o actividades que involucren aspectos económicos, científicos, resaltar sobre la importancia de la vegetación y su entorno cultural-natural en la vida del hombre e incrementar los espacios lúdico-recreativos dentro del Jardín Botánico-UCAJN.

Este análisis fue soportado con los mapas conceptuales integradores de cada fase para las dos instituciones educativas, producto de la revisión de cada mapa conceptual con el propósito de evaluar la pertinencia de la información suministrada, la evolución en el aprendizaje y la reestructuración de cada una de las charlas. En el mapa integrador de la fase de exploración se reflejan percepciones y preceptos generales y superficiales, resultado de la observación deliberada del entorno, Jardín Botánico-UCAJN, por parte de los niños (Figura 4); en el mapa obtenido de la fase de validación se observan conceptos más profundos directamente relacionados con los conocimientos brindados evidenciado en la mayor complejidad del mismo, producto de una información preparada y adecuadamente suministrada (Figura 5); y finalmente, en el mapa integrador de la fase de aplicación se refleja la pertinencia de contenidos y conceptos conectados con la realidad concreta y específica del entorno observado por los niños (Figura 6).

Tabla 1. Evaluación y valoración de mapas conceptuales de los estudiantes de la institución educativa CNFJCF, durante las tres fases.

\begin{tabular}{|l|l|l|l|l|l|l|l|l|l|l|l|}
\hline Fases & \multicolumn{4}{|l|}{ Evaluación } & \multicolumn{6}{l|}{ Valoración } \\
\cline { 2 - 12 } & NJ & RV & RC & EjA & E & EC & EC & H & M & R & C \\
\hline Exploración & 4,8 & 8,7 & 0 & 1,3 & 0,7 & 0,7 & 1 & 1 & 0,3 & 0,2 & 0 \\
\hline Validación & 5,1 & 9,7 & 1,2 & 1,6 & 0,9 & 0,7 & 0,9 & 0,6 & 0,8 & 0 & 0,2 \\
\hline Aplicación & 9,7 & 14,3 & 5,4 & 1,4 & 0,9 & 1 & 0,7 & 0,8 & 0,9 & 0,4 & 0,8 \\
\hline
\end{tabular}

E: Estético, EC: Ecológico, EC': Económico,

H: Humanístico, M: Moral, R: Recreativo, C: Cultural.

Tabla 2. Evaluación y valoración de mapas conceptuales de los estudiantes de la institución educativa RP, durante las tres fases.

\begin{tabular}{|c|c|c|c|c|c|c|c|c|c|c|c|}
\hline \multirow[t]{2}{*}{ Fases } & \multicolumn{4}{|c|}{ Evaluación } & \multicolumn{7}{|c|}{ Valoración } \\
\hline & $\mathrm{NJ}$ & RV & $\mathrm{RC}$ & EjA & $E$ & $\mathrm{EC}$ & $\mathrm{EC}^{\prime}$ & $\mathrm{H}$ & $\mathrm{M}$ & $\mathrm{R}$ & $\mathrm{C}$ \\
\hline Exploración & 5,9 & 6,4 & 0,4 & 1 & 0,6 & 0,7 & 0,7 & 0 & 0,1 & 0,1 & 0 \\
\hline Validación & 6,7 & 12,4 & 2,3 & 2,7 & 0,9 & 0,9 & 0,8 & 0,7 & 0,8 & 0,5 & 0,7 \\
\hline Aplicación & 9,7 & 14,8 & 9,7 & 1 & 1 & 1 & 0,6 & 0,9 & 0,9 & 0,8 & 0,6 \\
\hline
\end{tabular}

E: Estético, EC: Ecológico, EC': Económico, H: Humanístico, M: Moral, R: Recreativo, C: Cultural. 
Figura 3. Valoración mapas conceptuales.

Institución educativa CNFJCF

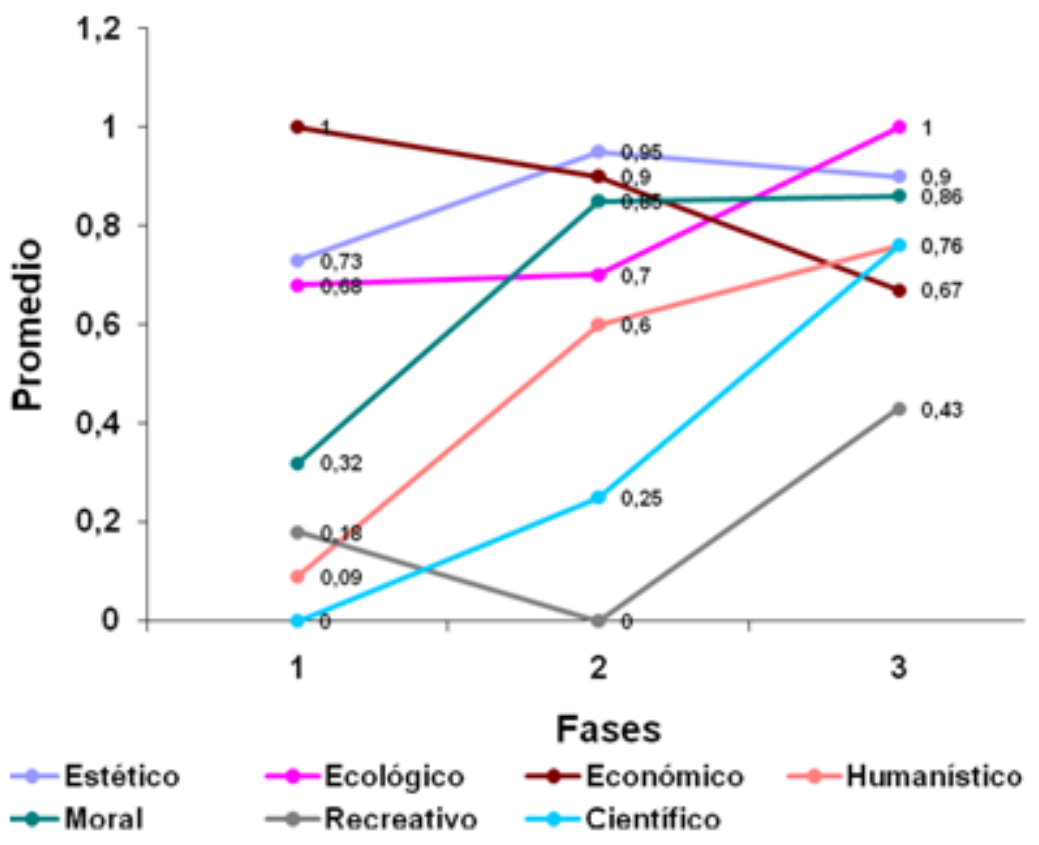

Institución Educativa RP

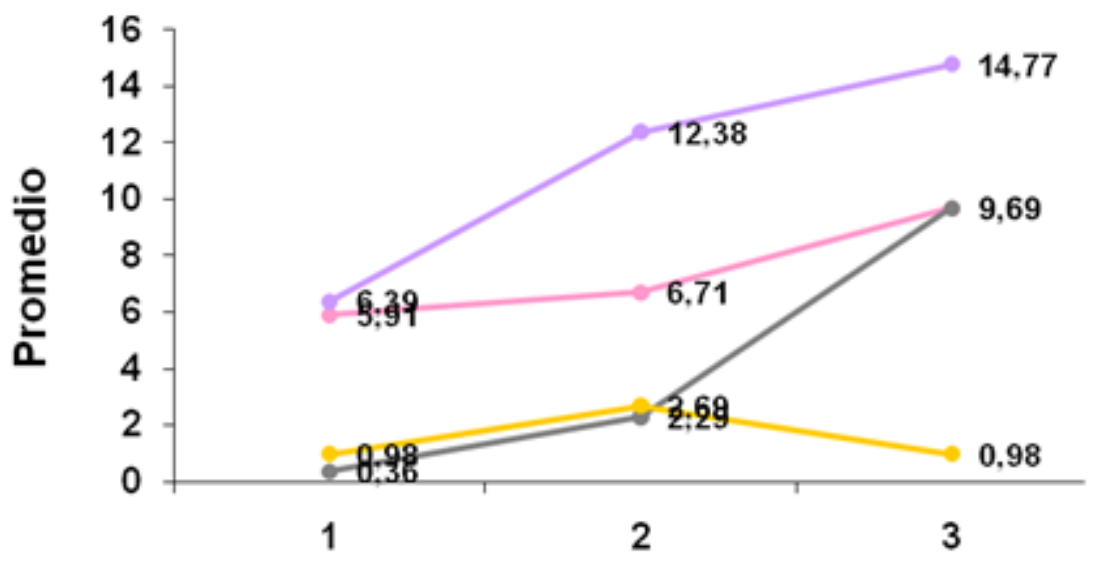

Fases

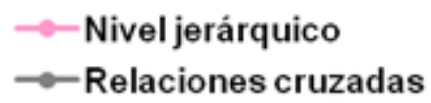

$\multimap$ Relaciones válidas
$\multimap$ Ejemplos adecuados

2) Charla modelo de interpretación ambiental

La progresión del aprendizaje significativo permitió la consolidación de la charla de interpretación, incluyendo los valores y conceptos necesarios para establecer un contenido profundo acorde con los recursos del entorno y capaces de conducir una experiencia vivificante, emocionante y creativa para los visitantes a través del recorrido. Como el medio ambiente de cualquier ecosistema está en constante cambio, la charla se consolida como modelo para futuros recorridos con escolares, 
supeditada a continuos cambios y renovaciones que permitan conducir experiencias directas e impactantes para este tipo de potenciales usuarios.

\section{DISCUSIÓN}

La estrategia interpretativa mediante tres fases: exploración, validación y aplicación, permitió consolidar una charla interpretativa modelo para niños en el Jardín Botánico-UCAJN gracias a la evaluación mediante la técnica de mapas conceptuales y aprendizaje significativo (Moneira, 2005; Cañas et al., n.f.); dada la importancia de los niños en los jardines botánicos de Colombia por sus amplios porcentajes de visita según los estudios de Macías, Sánchez y Ramírez (1999) y Sierra (2006).

El análisis interpretativo con niños en el Jardín Botánico-UCAJN, realizó un seguimiento completo y transversal al aprendizaje significativo adquirido por un grupo de niños de dos instituciones educativas (CNFJCF y RP) a través de las tres fases, a diferencia del estudio realizado por Macías et al. (1996) donde la estrategia interpretativa fue puesta a prueba con tres instituciones educativas diferentes en cada una de las fases, que no permite evidenciar cambios en un grupo específico.

\section{Fase de exploración}

La ausencia de una charla interpretativa soportada en el recurso, llevó a que la valoración de los mapas conceptuales no reflejara notoriamente los valores humanístico, moral, científico y recreativo; de modo que los niños centraron su capacidad perceptiva, entendimiento, desarrollo afectivo y emocional hacia los valores ecológico, económico y estético del ambiente circundante. En el mismo sentido, la evaluación indicó una deficiencia de aprendizaje significativo porque existió un promedio de 5,3 niveles jerárquicos, 7,5 relaciones válidas, 0,2 relaciones cruzadas, 1,1 ejemplos adecuados, por mapa conceptual para las dos instituciones educativas. El estudio de Macías et al. (1996) para esta misma fase, presentó valores promedio similares en la evaluación de mapas conceptuales sobre el Jardín Botánico del Quindío elaborados por los niños de la escuela urbana de Calarcá. En los dos trabajos estos resultados condujeron a mejorar la charla tratando de integrar todos los valores éticos, y contribuir con el aprendizaje significativo del estudiante para que sea capaz de replicar los conocimientos adquiridos en su vida cotidiana, fortaleciendo así la conciencia ambiental.

\section{Fase de validación}

La valoración de los mapas conceptuales indicó una mayor incidencia de valores ético-ambientales tales como: estético, económico, moral, ecológico y humanístico, siendo limitados los valores científico y recreativo para las dos instituciones educativas estudiadas. En la evaluación, los valores promedio obtenidos fueron: 5,9 niveles jerárquicos; 11,6 relaciones válidas; 1,7 relaciones cruzadas; 2,2 ejemplos adecuados, para las dos instituciones educativas. Lo anterior, evidenció que la información suministrada en la charla interpretativa logró captar el interés de los participantes en los espacios interpretados del Jardín Botánico-UCAJN, aumentando su capacidad cognoscitiva y la adquisición de una amplia gama de valores ético-ambientales que propenden por la conservación; las falencias encontradas fueron incluidas en la estructuración de la nueva charla interpretativa para la fase subsiguiente.

\section{Fase de aplicación}

La valoración de los mapas conceptuales, reflejó la más completa adquisición de valores éticoambientales por parte de los niños con referencia a las fases anteriores, aunque se presenta una leve disminución del valor económico entre las fases de validación y aplicación en las dos instituciones educativas. La evaluación indicó un progreso significativo en el aprendizaje, porque la calificación de los criterios incrementó con respecto a las etapas anteriores de la siguiente manera: 9,7 niveles jerárquicos; 14,5 relaciones válidas; 7,6 relaciones cruzadas, para las dos instituciones educativas, aunque ejemplos adecuados $(1,2)$ disminuyó con referencia a la fase anterior. Las disminuciones en los aspectos de valoración (económico) y evaluación (ejemplos adecuados) fueron soportadas con ideas y conceptos del entorno inmediato y la realidad cotidiana de los niños en las correcciones finales de la charla de esta fase, para consolidar la charla modelo de interpretación para niños dentro del Jardín Botánico-UCAJN. En Macías et al. (1996) participaron otras instituciones del área urbana (3) y rural (5), diferentes a las instituciones de las fases anteriores, profundizando en las temáticas de las estaciones que finalmente constituyeron el sendero en el Jardín Botánico del Quindío. De esta manera, al establecer comparaciones entre las temáticas se notó un progreso en el aprendizaje significativo y en la adquisición de valores ético-ambientales con 
referencia a las otras instituciones en las anteriores fases, lo que conllevó a catalogar la charla como base para realizar actividades interpretativas para niños.

\section{Seguimiento transversal del análisis interpretativo}

A través de las diferentes fases (exploración, validación y aplicación) se pudo evidenciar de forma general el incremento del aprendizaje significativo, la asimilación y la adquisición de la totalidad de los valores ético-ambientales por parte de los niños, afianzando el modelo constructivista de los procesos cognitivos humanos (Cañas et al., 2000) como se indica en la síntesis de la evaluación y valoración de mapas conceptuales para las instituciones educativas CNFJCF y RP (Figuras 2 y 3 ). Arbea y Del Campo (2004), en un estudio similar de seguimiento del aprendizaje, evaluaron la realización de mapas conceptuales sobre el tema de la energía antes y después de una instrucción en una muestra de estudiantes del Instituto de Educación Secundaria Alhama de la localidad de Corella, España; encontrando una mejora significativa en los mapas posteriores respecto a varios indicadores de aprendizaje de los estudiantes, poniendo de manifiesto un aprendizaje significativo. Del mismo modo, el estudio de Knapp (1996) permitió construir un modelo del cambio de comportamiento en interpretación ambiental con niños, influenciado por tres categorías de variables que deben manifestarse progresivamente en los visitantes cuando un programa interpretativo resulta ser efectivo: la primera categoría son las metas de inicio donde prevalece una conciencia, entendimiento y sensibilidad ambiental por el sitio; la segunda son las metas de posesión donde el usuario crea conciencia, investiga y evalúa las oportunidades del recurso; y finalmente, la tercera son las metas de apropiación donde se reflejan acciones ambientales responsables para promover el espacio. De esta manera, determinó que se requiere una interpretación fuertemente soportada en las oportunidades que brinda el recurso para influenciar positivamente en el aprendizaje y las actuaciones de los niños en pro de la protección del medio ambiente, representada en este estudio por la charla modelo para futuras actividades interpretativas en el Jardín Botánico-UCAJN.

\section{Charla modelo de interpretación ambiental}

El producto final del análisis interpretativo fue la charla estructurada para futuras caminatas interpretativas con niños dentro del Jardín Botánico-UCAJN, soportada con conceptualizaciones profundas del medio circundante que interactúan directamente con la mente de los escolares para hacer del recorrido una experiencia exitosa, que conlleve a la valoración y apropiación por el recurso interpretado. Esta charla fue también vivificante y profundizadora, capaz de captar la percepción y promover el entendimiento de los niños de forma dinámica y eficaz hacia el entorno interpretado, en lo cual se coincide con Macías et al. (1996).

Finalmente, se coincide con O'Brien y Peace (2004) en el cambio positivo en la adquisición de conocimientos y actitudes hacia la conservación después de una visita a espacios naturales por parte de los estudiantes, pero de igual forma se sugiere la necesidad de mantener la dinámica y transformación de las actividades de interpretación ambiental y la cualificación de los intérpretes del lugar.

\section{CONCLUSIONES}

El estudio de interpretación ambiental con los niños de grados cuarto y quinto de las instituciones educativas CNFJCF y RP, permitió consolidar una charla modelo de I.A. para el Jardín BotánicoUCAJN dirigida a este tipo de visitantes, gracias a la identificación de sus conocimientos previos durante la fase de exploración y a la evolución del aprendizaje significativo en cuanto a la asimilación de conceptos biológicos y la adquisición integral de valores ético-ambientales a través de las fases de validación y aplicación.

La valoración y evaluación de mapas conceptuales no evidencian diferencias significativas respecto a la procedencia urbana o rural de los estudiantes, ni tampoco de las diferencias conceptuales entre ambas instituciones.

Resulta factor clave la formación y cualificación de intérpretes ambientales en jardines botánicos y especialmente con niños, que hagan sistematización y evaluación de los procesos interpretativos y que tengan en cuenta el reconocimiento de los conceptos previos y valores ético-ambientales de los visitantes, con el fin de generar construcción significativa y cambio de actitudes a favor de los ecosistemas y sus componentes. 


\section{AGRADECIMIENTOS}

A los niños y niñas, docentes y administrativos de las instituciones educativas: Comercial del Norte Francisco José Chaux Ferrer y Rafael Pombo, a la administración del proyecto Jardín BotánicoUCAJN y a sus operarios, y al Grupo de Estudios Ambientales (GEA) y a la Universidad del Cauca, por el apoyo para la realización de esta investigación.

\section{REFERENCIAS}

- Acevedo, María P. (2002). Expedición: Ciencias naturales y educación ambiental. Básica primaria $4^{\circ}$. Bogotá. Ed. Norma.

- Arbea, Javier, y Del Campo, Francisco. (2004). Mapas conceptuales y aprendizaje significativo de las ciencias naturales: análisis de los mapas conceptuales realizados antes y después de la implementación de un módulo instruccional sobre la energía. En A. J. Cañas, J.D. Novak, \& F. M. González (Eds.), Concept Maps: Theory, Methodology, Technology. Proc. of the First International Conference on Concept Mapping. Pamplona, España. Obtenido el 14 de diciembre de 2009, desde http://cmc.ihmc.us/papers/cmc2004148.pdf

- Cañas, Alberto, Kenneth, J. Ford, Patrick, M. Hayes, Reichherzer, Thomas, Suri, Niranjan, Coffey, John, Carff, Roger, y Hill, Greg.(n.f.) Colaboración en la construcción del conocimiento mediante mapas conceptuales. Institute for Human and Machine Cognition University of West Florida. Obtenido el 7 de diciembre de 2009, desde www.ihmc.us/users/acanas/ColabCon.pdf -

- Cañas, Alberto, Kenneth, J. Ford, Patrick, M. Hayes, Reichherzer, Thomas, Suri, Niranjan, Coffey, John, Carff, Roger, Hill, Greg, y Breedy, Maggie. (2000). Herramientas para construir y compartir modelos de conocimiento basados en mapas conceptuales. Revista de Informática Educativa, 13(2), 145-158. Institute for Human and Machine Cognition University of West Florida. Obtenido el 8 de diciembre de 2009, desde http://lidie.uniandes.edu.co/revista

- Dawson, L. (1999). Cómo Interpretar Recursos Naturales e Históricos. Costa Rica: WWF.

- Figueroa, Apolinar, Benítez, Cristina, Cabezas, Fabio, Zambrano, Leonidas, Ramírez, Bernardo, y Sanabria, Olga. (2002). Proyecto Jardín Botánico Álvaro José Negret. Grupo de Estudios Ambientales -GEA-. Popayán: Universidad del Cauca.

- Gómez, Miguel Ángel. (2002). El modelo tradicional de la pedagogía escolar: Orígenes y precursores. Revista de Ciencias Humanas, 28, 1-2. Pereira: UTP.

- Knapp, Doug. (1996). Evaluating the impact of environmental interpretation: a review of three research studies. Coalition for education in the outdoors research Symposium Proceedings [Bradford Woods-Indiana]. Obtenido el 3 de marzo de 2010, desde http://www.eric.ed.gov/ERICDocs/data/ericdocs2sql/ content_storage_01/0000019b/80/15/07/da.pdf. Knapp, Doug, y Benton, Gregory M. (2004). Elements to successful interpretation: a multiple case study of five national parks. Journal of Interpretation Research, 9(2). Obtenido el 31 de enero de 2010, desde http://www.interpnet.com/JIR/pdf/JIR-v9n2.pdf

- Macías, Diego; Sánchez, Liliana y Ramírez, Jair. (1996). Propuesta de educación ambiental para el Jardín Botánico del Quindío, dirigida a los estudiantes de básica primaria primaria del municipio de Calarcá, Quindío. Trabajo de Grado, Especialización en Educación Ambiental, Universidad del Quindío.

- Macías, Diego, Sánchez, Liliana, y Ramírez, Jair. (1999). Interpretación ambiental en el Jardín Botánico del Quindío. Revista de Investigaciones, 8, 30-47. Universidad del Quindío.

- Moneira, Marco. (2005). Mapas conceptuales y aprendizaje significativo en ciencias. Cadernos do Aplicacão, 11(2), 143-156. Porto Alegre, Brasil. Obtenido el 15 de diciembre de 2009, desde http://www.if.ufrgs.brl moreira/mapasesp.pdf

- Morales, Jorge. (1998). Guía práctica para la interpretación del patrimonio. El arte de acercar el legado natural y cultural al público visitante. Sevilla, España: Junta de Andalucía, Consejería de Cultura, Empresa Pública de Gestión de Programas Culturales.

- $\quad$. (n.f.). La planificación interpretativa asegura la excelencia en interpretación. Obtenido el 12 julio de 2010, desde http://www.interpretaciondelpatrimonio.com/docs/pdf/Planificacioninterpretativa.pdf

- Novak, J. D., y Gowin, D .B. (1988). Aprendiendo a aprender. Barcelona: Martínez Roca.

- O'Brien, Jason, y Peace, James. (2004). The Effects of a National Wildlife Refuge's EE Programs on Elementary School Classes' Knowledge and Attitudes. Journal of interpretation Research, 9(2), 27-45. 
- Psacharopoulos, Jorge, Rojas, Carlos, y Vélez, Eduardo. (1992). Achievement Evaluation of Colombia's Escuela Nueva. Is Multigrade the Answer? Technical Department. Latin American and the Caribbean Region. World Bank.Obtenido 2 de diciembre de 2009 desde http://www- wds. worldbank.org /servlet/ WDSContentServer/ WDSP/ IB/ 1992/04/01/000009265_3961002224506/ Rendered/PDF/multi_page.pdf

- Samper, Cristián, y García, Hernando. (2001a). Estrategia Nacional para la Conservación de Plantas. Bogotá: Instituto Alexander von Humboldt, Red Nacional de Jardines Botánicos, Ministerio del Medio Ambiente, Asociación Colombiana de Herbarios.

$\bullet$ (2001b). Plan Nacional de Jardines Botánicos. Bogotá: Instituto Alexander von Humboldt, Red Nacional de Jardines Botánicos. Ministerio del Medio Ambiente, Botanic Gardens Conservation International, Darwin Initiative.

- Sierra, Paola. (2004). Educación ambiental no formal e informal acerca de la educación ambiental en los jardines botánicos de Colombia: experiencias educativas en red. Encuentro de experiencias educativas en educación ambiental, Pontificia Universidad Javeriana, Bogotá.

- - (2006). Educación, ambiente y niñez: experiencia en red de los jardines botánicos de Colombia. Bogotá: La Red.

- Talero, Elsa, y Umaña, Gloria. (1993). Educación ambiental: Capacitación de docentes de básica primaria. Bogotá: Codesa.

- Tilden, Freeman. (1957). Interpreting our heritage. Chapel Hill: The University of Nort Carolina Press.

- Torres, Rosa María. (1992). Alternativas dentro de la educación formal: el programa escuela nueva de Colombia. Perspectivas, 84. París: UNESCO. Obtenido el 9 de febrero de 2010, desde http://www.oei.es/equidad/esnueva.PDF

1. Bióloga Universidad del Cauca. Estudiante Tecnología en Medio ambiente, Santa Fe Community College, Santa Fe, NM USA. macruz258@gmail.com

2. Bióloga Universidad del Cauca. biologia96@hotmail.com

3. Licenciado en Biología y Educación Ambiental. Especialista en Educación Ambiental. Magíster en Conservación y Gestión del Medio Natural. Profesor Titular, Departamento de Biología, Universidad del Cauca. Grupo de estudios sobre diversidad vegetal - Sachawaira

Flgura 4. Mapa conceptual Integrajo para las dos instivelones educativas. Fase de exploracion.

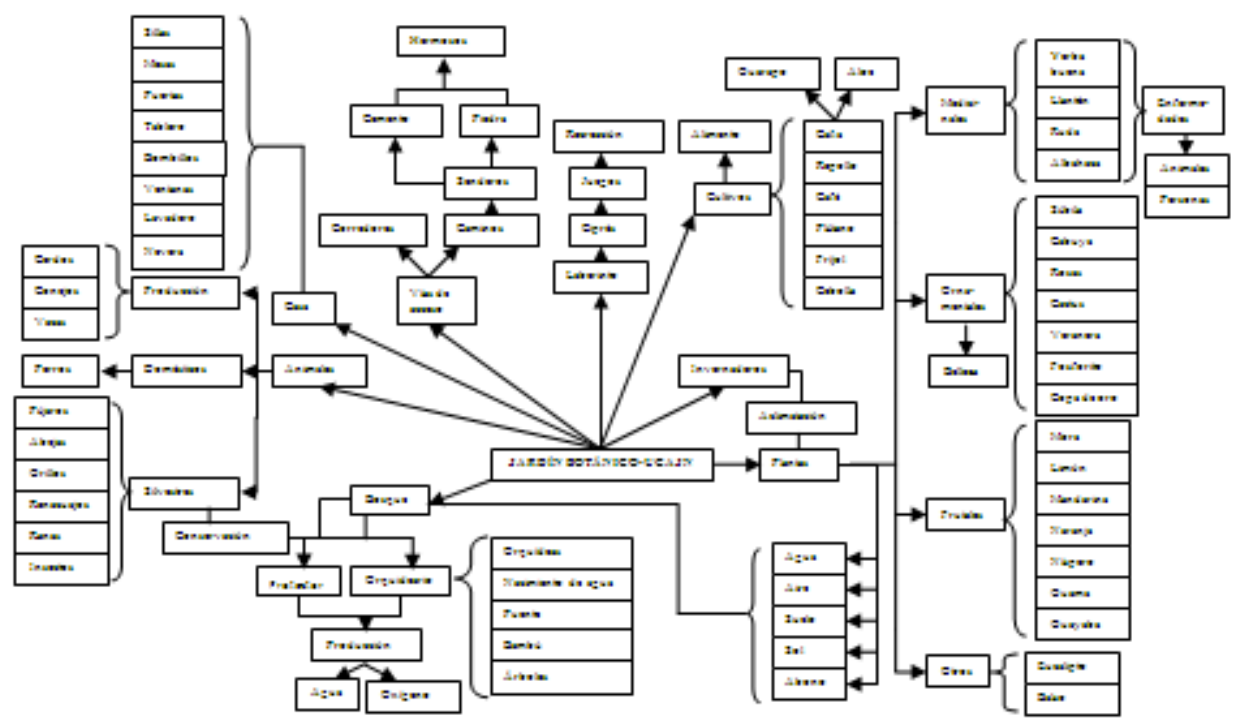




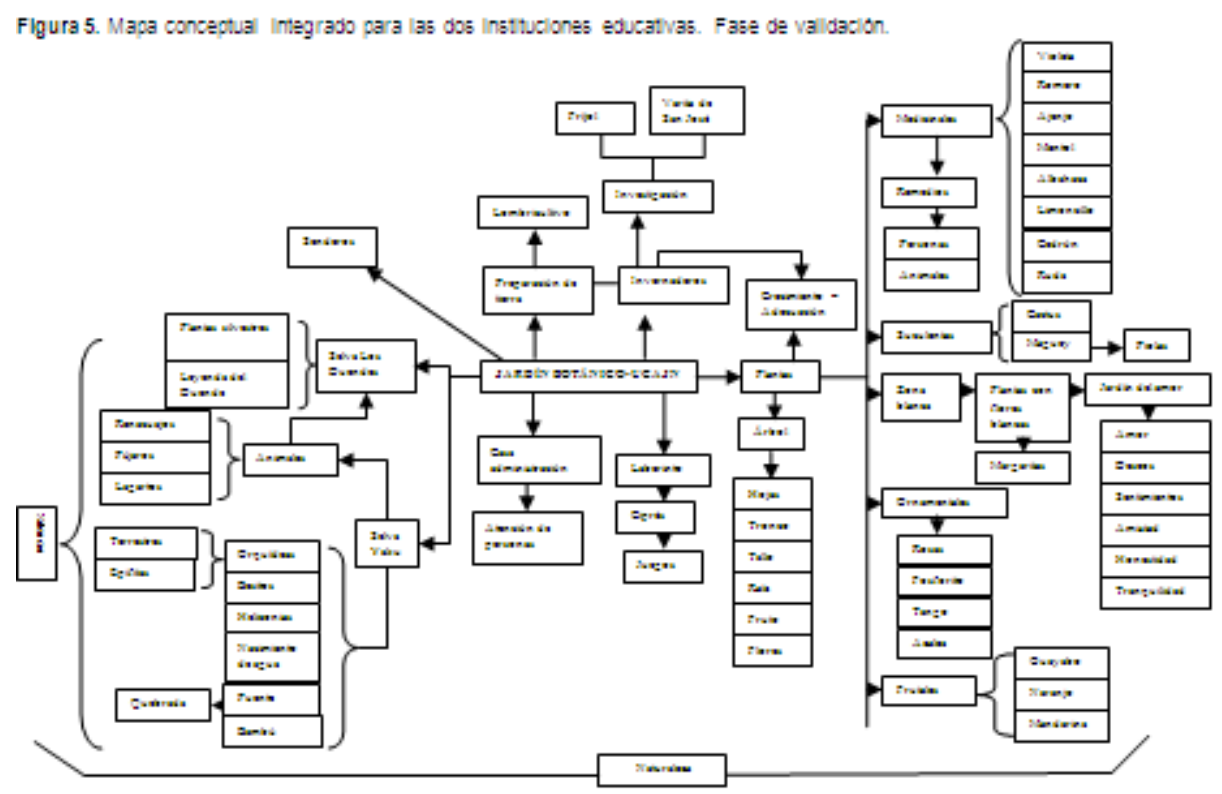

Flgura 6. Mapa conceptual integrajo para las dos instivelones educativas. Fase de aplicacion.

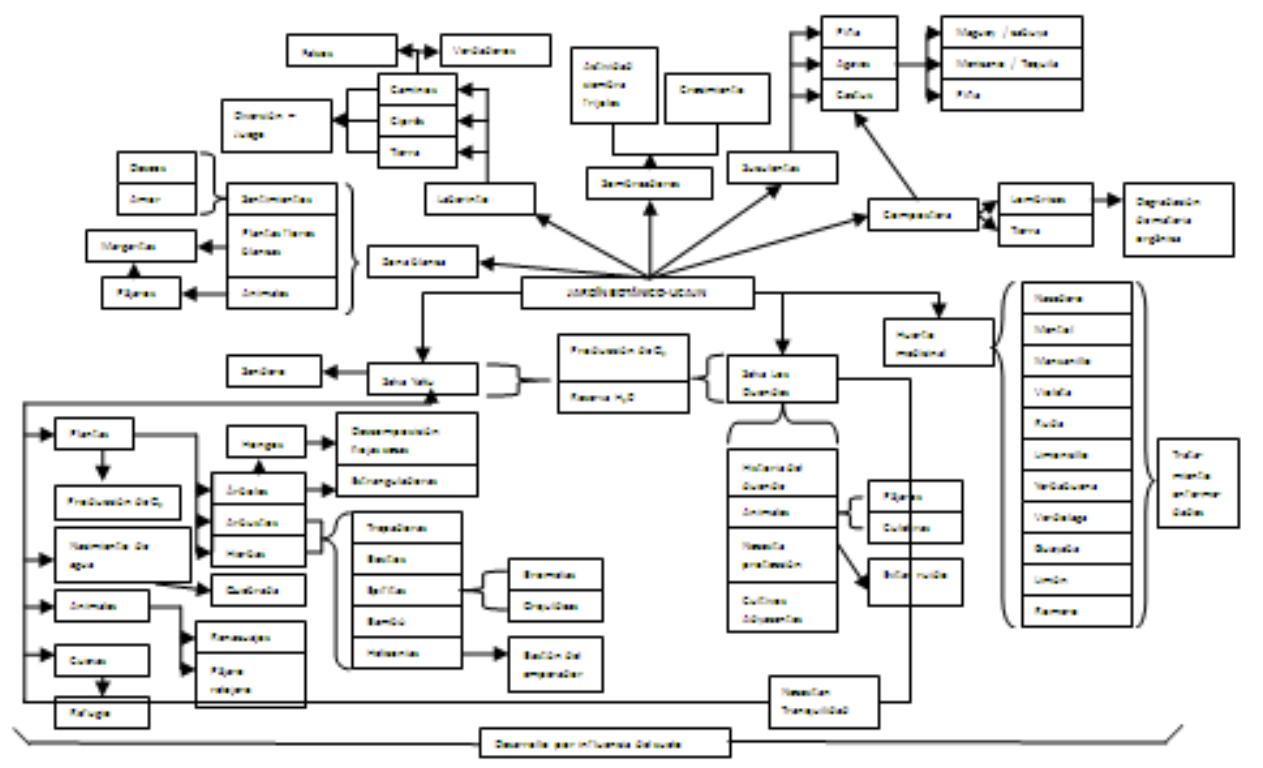

